\title{
Factors Associated With Emergency Department Discharge, Outcomes and Follow-Up Rates of Stable Patients With Lower Gastrointestinal Bleeding
}

\author{
Tracey A. Martin ${ }^{\mathrm{a}, \mathrm{e}}$, Sunena Tewani ${ }^{\mathrm{b}}$, Lindsay Clarkec, Aiya Aboubakrc, Srikanth Palanisamyc, \\ Jihui Leed ${ }^{\mathrm{d}}$, Carl V. Crawford ${ }^{\mathrm{a}}$, David W. Wan ${ }^{\mathrm{a}}$
}

\begin{abstract}
Background: Lower gastrointestinal bleeding (LGIB) is a common reason for hospitalization. However, recent data suggest low-risk patients may be safely evaluated as an outpatient. Here, we compare stable LGIB patients discharged from the emergency department (ED) with those admitted, determine factors associated with discharge and 30-day outcomes, and evaluate follow-up rates amongst the discharged cohort.

Methods: A retrospective study of stable LGIB patients (heart rate $<100$ beats/min, systolic blood pressure $>100 \mathrm{~mm} \mathrm{Hg}$ and blood on rectal exam) who presented to the ED was conducted. Factors associated with discharge and rates of outpatient follow-up were determined in the discharged cohort. Therapeutic interventions and 30-day outcomes (including re-bleeding, re-admission and mortality rates) were compared between the admitted and discharged groups.

Results: Ninety-seven stable LGIB patients were reviewed, of whom $38 \%$ were discharged and characteristics associated with discharge included age $(\mathrm{P}<0.001)$, lack of aspirin $(\mathrm{P}<0.002)$ and anticoagulant $(\mathrm{P}<0.004)$ use, higher index hemoglobin $(\mathrm{P}<0.001)$ and albumin $(\mathrm{P}<0.001)$, lower blood urea nitrogen $(\mathrm{P}<0.001)$ and creatinine $(\mathrm{P}=0.008)$, lower Oakland score $(\mathrm{P}<0.001)$, lower Charlson Comorbidity Index $(\mathrm{P}<0.001)$ and lack of transfusion requirements $(\mathrm{P}$ $<0.001)$. There was no statistical difference in 30-day re-bleeding, re-admission or mortality rates between admitted and discharged patients. Discharged patients had a $46 \%$ outpatient follow-up rate.
\end{abstract}

Manuscript submitted June 2, 2021, accepted July 6, 2021

Published online July 28, 2021

aDivision of Gastroenterology and Hepatology, Weill Cornell Medicine, New York-Presbyterian Hospital, New York, NY, USA

bDivision of Hospital Medicine, Weill Cornell Medicine, New York-Presbyterian Hospital, New York, NY, USA

'Department of Medicine, Weill Cornell Medicine, New York-Presbyterian Hospital, New York, NY, USA

${ }^{\mathrm{d}}$ Department of Population Health Sciences, Weill Cornell Medicine, New York, NY, USA

${ }^{\mathrm{e} C}$ Corresponding Author: Tracey Martin, Weill Cornell Medicine, 1305 York Avenue, Fourth Floor, New York, NY 10021, USA.

Email:tm269@cornell.edu

doi: https://doi.org/10.14740/gr1425
Conclusions: While early discharge in low-risk LGIB patients appears to be safe and associated with a decrease in length of stay, further studies are needed to guide timely and appropriate outpatient evaluation.

Keywords: Colonoscopy; Hematochezia; Lower gastrointestinal bleed; Oakland score; Rectal bleeding

\section{Introduction}

Lower gastrointestinal bleeding (LGIB) accounts for about $30 \%$ of hospitalizations related to gastrointestinal (GI) hemorrhage [1]. Bleeding can range from low volume to substantial blood loss causing hemodynamic instability. The American College of Gastroenterology (ACG) guidelines for LGIB recommend colonoscopy as the first-line test for both low-risk and high-risk patients; however, the optimal timing of colonoscopy remains unclear [2]. Studies have demonstrated that colonoscopies performed early (within $24 \mathrm{~h}$ ) rarely result in definitive diagnoses, therapeutic intervention, or improved clinical outcomes when compared to non-urgent colonoscopies (> 24 h) [3-5]. If early colonoscopy does not appear to provide clinical benefits and non-urgent colonoscopies generally yield little information, the natural question is whether stable low-risk patients with LGIB even benefit from inpatient colonoscopies, particularly as this often leads to an extended length of stay (LOS). As a result, it may be that outpatient management would be a more cost-effective approach. Though not fully studied, we predict that outpatient management of low-risk LGIB is likely safe, feasible, and potentially cost-effective.

While there have been a few recent studies evaluating safe and early discharge in low-risk patients, there are still no clear practice guidelines in the United States of America (USA) on risk stratification to determine safety for discharge from the emergency department (ED) as well as optimal timing of outpatient evaluation. A study in Germany by Kheng-Seong et al demonstrated that of $53 \%$ of patients admitted with LGIB, only $35 \%$ underwent lower endoscopy and only $4 \%$ had therapeutic intervention [6]. While this study further supported the limited utility of inpatient colonoscopies outcomes and follow- 
up data for those discharged from the ED were not specified. In the United Kingdom (UK), Oakland et al developed a risk score to identify patients with LGIB that could be safely discharged for outpatient workup [7]. The Oakland score includes age, gender, prior history of GI bleeding, heart rate (HR), systolic blood pressure (SBP) and hemoglobin ( $\mathrm{Hgb})$ and predicts a $95 \%$ probability of safe discharge for a score $\leq 8$ [7]. As such, the 2019 British Society of Gastroenterologists guideline on LGIB now recommends that patients identified as lowrisk should be discharged from the ED to avoid admission [8]. While this study included 28-day re-admission and mortality rates, it did not differentiate low-risk from high-risk patients and outcomes and outpatient follow-up in those discharged from the ED were not specified. A recent study in the USA by Oakland et al demonstrated that an even higher threshold with an Oakland score of 10 was predictive of safe discharge; however, no clear guidelines on timing and indication for postdischarge evaluation were described [9].

Guidelines and recommendations for healthcare systems to identify low-risk patients who can be safely discharged with a mechanism in place for timely outpatient follow-up have yet to be established. While diverticulosis, hemorrhoids, and angiodysplasia are some of the most common etiologies of LGIB, some patients may need further evaluation to rule out etiologies such as malignancy and inflammatory bowel disease. In this study, our primary aim was to determine factors associated with discharge from the ED at a large, tertiary hospital in the USA. Our secondary aim was to compare outcomes between admitted and discharged patients, as well as evaluate followup amongst the discharged cohort given the importance of outpatient evaluation to rule out more serious etiologies.

\section{Materials and Methods}

\section{Database}

This was a retrospective cohort study that utilized the Informatics for Integrating Biology and the Bedside software to generate a database of LGIB patients presenting to the ED at an academic tertiary care center in New York City, USA, between November 1, 2018 and October 31, 2019. In our electronic medical record system, patients with a diagnosis of LGIB were identified. LGIB was defined by ICD-10 codes for GI hemorrhage unspecified, hemorrhage of the anus and rectum, and diverticular disease of the small intestine without perforation or abscess (ICD 10 codes: K92.2, K62.5, K57.1). This study was approved by the Institutional Review Board at Weill Cornell Medicine. This study was conducted in compliance with the ethical standards of the responsible institution on human subjects as well as with the Helsinki Declaration.

Of the 129 patients reviewed, 97 met inclusion criteria including a chief complaint and primary diagnosis of rectal bleeding, clinical evidence of LGIB defined as visible hematochezia or maroon stools on rectal exam. Twenty-five patients were excluded due to hemodynamic instability defined by an $\mathrm{SBP}<100 \mathrm{~mm} \mathrm{Hg}$ or HR $>100$ beats/min on presentation. Patients younger than 18 years of age, without maroon stools or hematochezia on rectal exam, pregnant, had polypectomy within 30 days, with signs/symptoms of suspected upper GI source or with signs of hemodynamic instability were excluded. Data were collected on patient demographics, clinical features, comorbidities, medications, hemodynamic parameters, laboratory values, and diagnostic imaging. Findings and therapeutic outcomes for GI luminal evaluation with esophagogastroduodenoscopy (EGD), colonoscopy, or flexible sigmoidoscopy were noted. The Oakland score, a risk-assessment tool for safe discharge in LGIB and the Charlson Comorbidity Index (CCI), a score based on combinations of comorbid condition to predict 1-year mortality risk, were calculated $[7,10]$.

Admission or ED discharge status was also obtained on each patient along with outpatient follow-up plan provided by the ED, show rate and specialty provider seen. Patients seen by outpatient providers outside our electronic medical recording system were excluded from outcome analysis due to inability to access patient charts. Reasons commonly cited for admission was visible blood on rectal exam, use of blood thinners, $\mathrm{Hgb}$ drop and continued monitoring. Reasons for discharge included stable Hgb, stable hemodynamics, bleeding resolved and patient's preference.

\section{Outcome measures}

The primary objectives were to compare the proportion of patients with stable overt LGIB that were discharged from the ED to those admitted, and identify factors associated with discharge. Secondary objectives were to compare rate of therapeutic interventions, LOS, 30-day recurrence of bleeding (defined as bleeding occurring after $24 \mathrm{~h}$ of cessation of index bleed), thrombosis, and mortality rates between the discharged and admitted group. In the discharge cohort, the ED followup mechanism was evaluated by determining the proportion of patients with follow-up compared to those lost to followup, the type of specialty provider seen on discharge, timing of follow-up, and assessing for any distinct characteristics among those that followed up versus those that did not. While the decision for discharge included personal subjectivity and clinical judgement of the provider, we aimed to model the pattern of association of discharge status with demographic and clinical characteristics and identify statistically significant predictors of discharge status.

\section{Statistical analysis}

Descriptive statistics were reported for demographic clinical features; median and interquartile range (IQR) were calculated for a numeric variable and frequency and percentage were calculated for a categorical variable. The Wilcoxon rank-sum test for admission status and Kruskal-Wallis test for followup status were used for comparing numeric demographic and clinical features. The Fisher's exact test was used for comparing categorical demographic and clinical features. A two-sided P-value less than 0.05 was considered statistically significant. Logistic regression models were fitted to model the effects of 
the predictors on discharge status. Unadjusted logistic regression models estimated the crude odds ratio (OR) of getting discharged, while adjusted logistic regression models estimated the conditional effect of the predictor on the odds of getting discharged controlling for age, race, and gender. The OR and its $95 \%$ confidence interval (CI) were reported. All statistical analyses were conducted using $\mathrm{R}$ software version 3.6.1 (R Core Team, Vienna, Austria).

\section{Results}

\section{Discharged and admitted groups}

A total of 97 stable patients presenting to the ED with overt LGIB on rectal exam were evaluated, including 59 males and 38 females, with a median age of 70 years. Baseline characteristics are shown in Table 1 . Overall, $28 \%$ of patients had a prior history of GIB, 31\% were on aspirin (ASA) and 20\% were on a direct oral anticoagulant (DOAC) or warfarin. Eighty-one patients presented with bright red blood on rectal exam and 16 patients had maroon stools. The median HR was 82 beats/min, SBP was $134 \mathrm{~mm} \mathrm{Hg}$, median index $\mathrm{Hgb}$ was $12.5 \mathrm{~g} / \mathrm{dL}$ and the median international normalized ratio (INR) was 1.1 (Table 1). Overall, 60 (62\%) patients were admitted and $37(38 \%)$ patients were discharged from the ED.

\section{Investigation and interventions}

Out of 97 patients seen in the ED, 46 patients had a GI consult, 43 of which were admitted with most of the consults occurring after admission. Among the 60 patients admitted, 29 (48\%) patients underwent colonoscopies or flexible sigmoidoscopies (27 colonoscopies and two flexible sigmoidoscopies) during their admission. Of those discharged, seven (19\%) patients had colonoscopies or flexible sigmoidoscopy (six colonoscopies and one flexible sigmoidoscopy), five of which were done as an outpatient and two of which occurred after re-admission for GIB. The most common findings were diverticulosis $(\mathrm{n}=$ $13)$, hemorrhoids $(n=7)$, rectal/colonic ulcer $(n=6)$ and angiodysplasia $(n=3)$ (Table 2$)$. A total of six $(17 \%)$ patients who underwent colonoscopy had a therapeutic intervention with hemostatic clips or argon plasma coagulation (APC), all of whom were admitted and accounted for $6 \%$ of the overall cohort (Table 2). Eleven admitted patients had a computed tomography angiography (CTA), three of which were positive resulting in two patients undergoing interventional radiologyguided angiography for an angiodysplasia and a bleeding rectal ulcer with a visible vessel. No surgical intervention was performed in this cohort.

\section{Clinical course of discharged group}

For all discharged patients follow-up was assessed by reviewing the ED's disposition notes and discharged instructions. Of 37 patients, three patients were given an appointment prior to discharge, seven patients were later contacted with a follow-up appointment by a patient navigator or social worker, 26 patients were responsible for making their own appointment and one patient had no designated follow-up plan listed. Within this cohort, 17 patients did follow up post-ED discharge for GIB with either a primary care provider (PCP), gastroenterologist or colorectal surgeon, eight patients did not follow up with one of these providers for bleeding, and 12 patients had no follow-up information within our medical recording system. Of the 17 patients that did follow up, 65\% were responsible for making their own appointments. The three patients that had appointments given to them prior to discharge followed up as an outpatient. There was no statistically significant difference in age, race or gender when comparing the patients that did follow up post-discharge compared to those that did not (Table 3).

\section{Comparison of outcomes between discharged and admit- ted groups}

Overall, 19 patients required blood transfusions with index Hgb ranging from 6.2 to $13.1 \mathrm{~g} / \mathrm{dL}$, all of whom were admitted. Six percent required intensive care unit (ICU) stay. Outcomes at 30 days were assessed in $70 \%$ of patients with an average lost to follow-up rate of about $31 \%$ (27\% of admitted and $41 \%$ of discharged patients). The 7-day and 30-day re-bleeding rates for the admitted group were $3.3 \%$ and $7 \%$, respectively, compared to $8 \%$ and $14 \%$ among the discharged group. The 30 -day bleeding-related death was $1.7 \%$ in the admitted group compared to $0 \%$ in the discharged group with a lost to follow-up rate of $27 \%$ vs. $38 \%$ respectively. One admitted patient died related to LGIB, who was also in multiorgan failure and deemed too unstable for colonoscopy. The all-cause 30-day mortality rate in the admitted group compared to the discharged group was $7 \%$ vs. $0 \%$ respectively, with a lost to follow-up rate of $27 \%$ vs. $38 \%$ respectively. Patients discharged from the ED were evaluated for a median LOS of $5 \mathrm{~h}$ compared to a median of 2 days in those admitted to the hospital (Table 4).

\section{Distinctive characteristics between discharged vs. admit- ted LGIB patients}

Compared to those admitted, patients discharged from the ED were of younger age (53 vs. 74.5 years, $\mathrm{P}<0.001$ ), with a statistically significant lower CCI (2 vs. $5, \mathrm{P}<0.001)$ and a lower Oakland score (11 vs. 18.5, $\mathrm{P}<0.001)$ (Table 1$)$. The discharge cohort was less likely to be on antiplatelets and anticoagulants. Four discharged patients were on ASA compared to 26 in the admitted cohort $(\mathrm{P}=0.001)$, and no patients discharged were on a DOAC compared to 14 in the admitted group $(\mathrm{P}<0.004)$ (Table 1$)$. Though not statistically significant, one discharged patient was on warfarin compared to four in the admitted group $(\mathrm{P}=0.64)$ (Table 1$)$. Patients discharged had a higher median Hgb (13.9 vs. $10.6 \mathrm{~g} / \mathrm{dL}, \mathrm{P}<0.001)$ with the lowest Hgb amongst the discharged group being $9.3 \mathrm{~g} /$ $\mathrm{dL}$ compared to $3.4 \mathrm{~g} / \mathrm{dL}$ in those admitted. $\mathrm{Hgb}<10 \mathrm{~g} / \mathrm{dL}$ 
Table 1. Demographics and Characteristics of All Patients Presenting to the ER With Stable LGIB

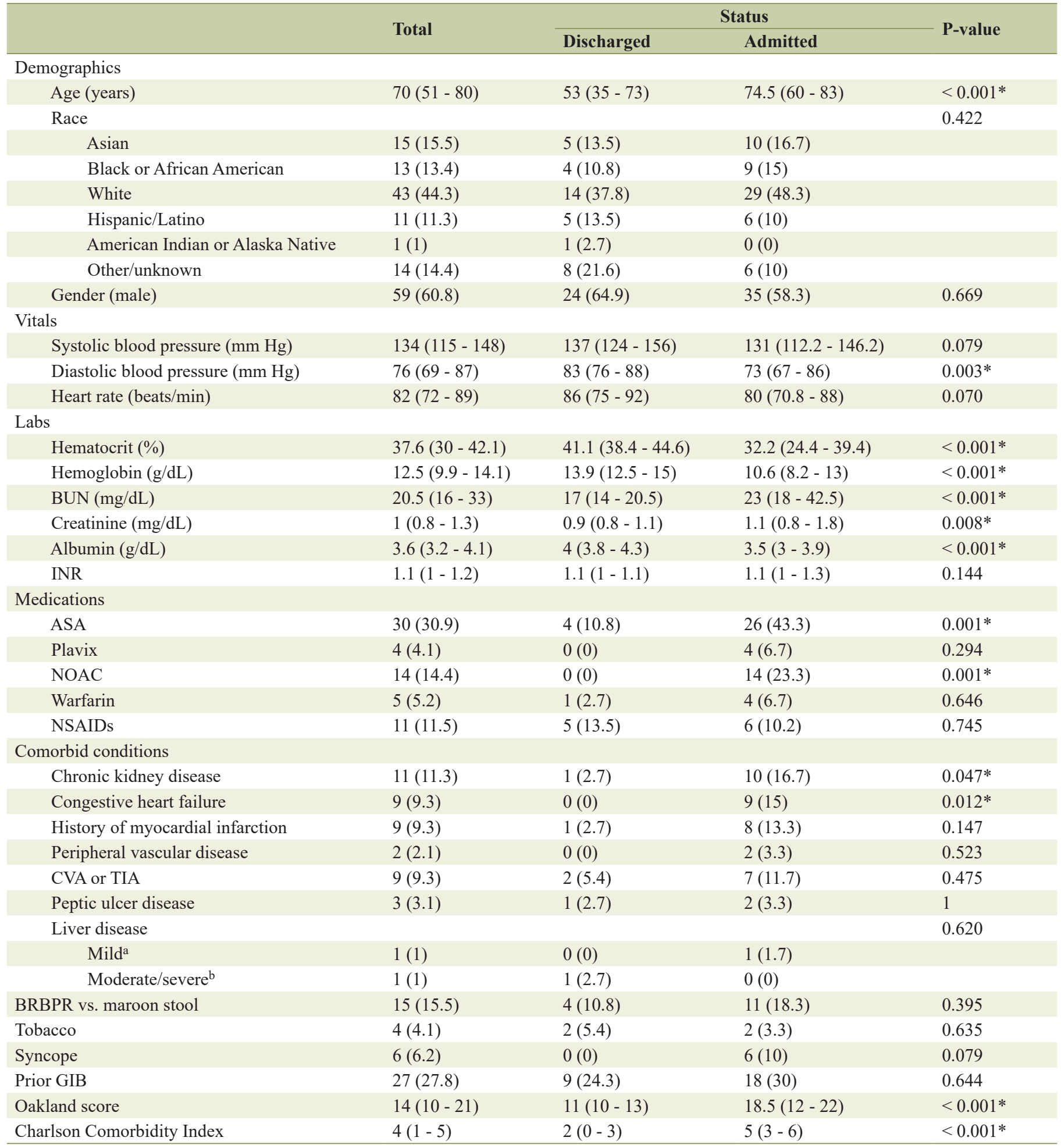

Data are presented as frequency (\%) or median (IQR). P-value is calculated from a Wilcoxon rank-sum test for a numeric variable and a Fisher's

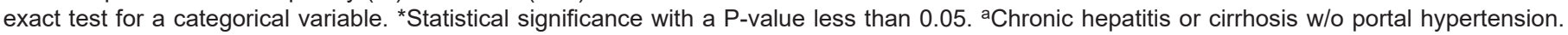
${ }^{b}$ Cirrhosis and portal hypertension w/wo variceal bleed. LGIB: lower gastrointestinal bleeding; ED: emergency department; IQR: interquartile range; BUN: blood urea nitrogen; GIB: gastrointestinal bleeding; DM: diabetes mellitus; INR: international normalized ratio; ASA: aspirin; DOAC: direct oral anticoagulant; NSAIDs: nonsteroidal anti-inflammatory drugs; BRBPR: bright red blood per rectum; AIDS: acquired immunodeficiency syndrome; COPD: chronic obstructive pulmonary disease; CVA: cerebrovascular accident; TIA: transient ischemic attack. 
Table 2. Investigation and Interventions Amongst Discharged and Admitted Patients With LGIB

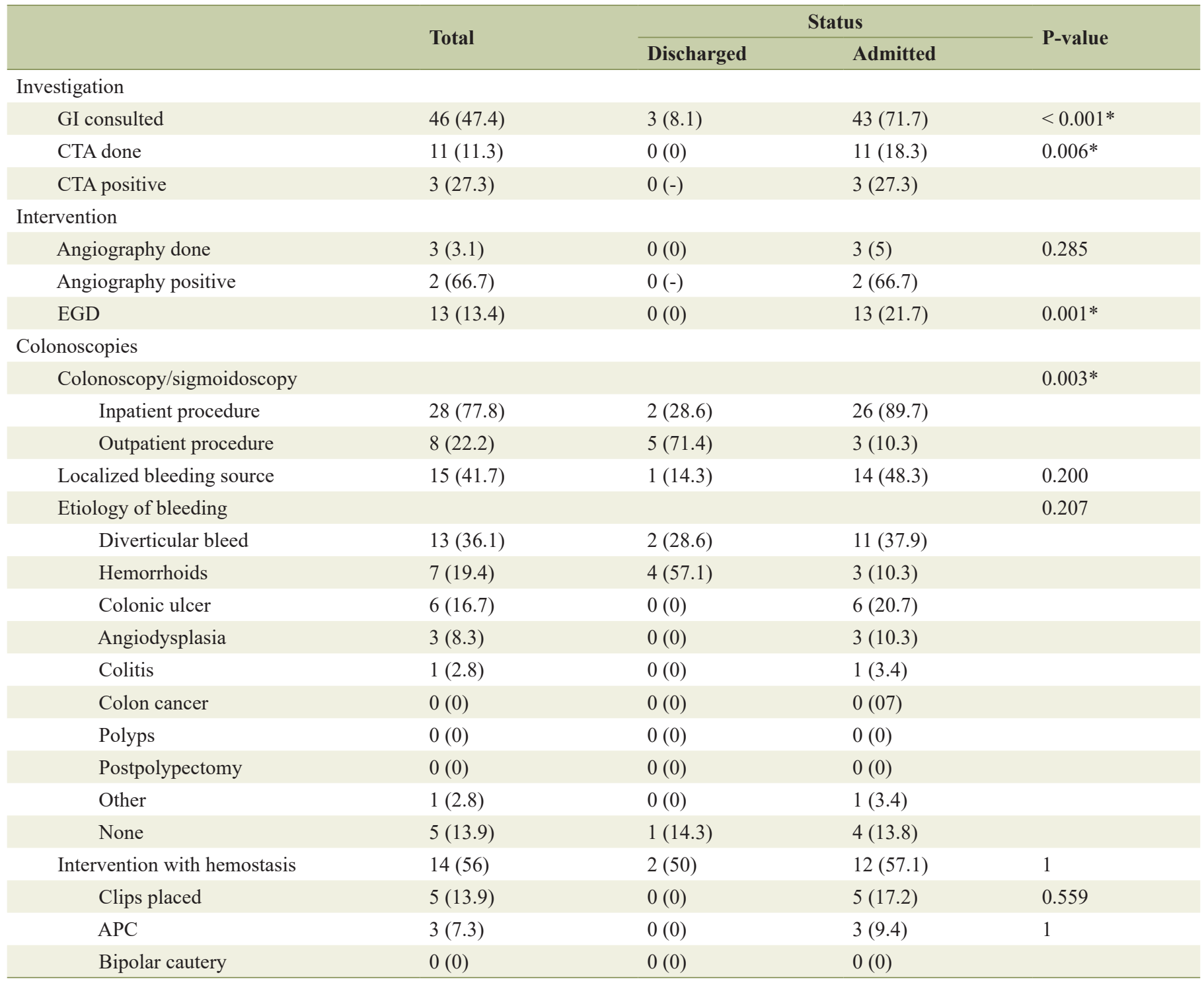

Data are presented as frequency (\%) or median (IQR). P-value is calculated from a Wilcoxon rank-sum test for a numeric variable and a Fisher's exact test for a categorical variable. *Statistical significance with a P-value less than 0.05. LGIB: lower gastrointestinal bleeding; GI: gastrointestinal; CTA: computed tomography angiography; EGD: esophagogastroduodenoscopy; APC: argon plasma coagulation.

was observed among $42 \%$ of admitted patients compared to $2.7 \%$ in the discharged group. The discharged group also had a lower median blood urea nitrogen (BUN, 17 vs. $23 \mathrm{mg} / \mathrm{dL}$, $\mathrm{P}<0.001)$ and creatinine $(\mathrm{Cr}, 0.9$ vs. $1.1 \mathrm{mg} / \mathrm{dL}, \mathrm{P}<0.008)$ and a higher median albumin level (4 vs. $3.5 \mathrm{~g} / \mathrm{dL}, \mathrm{P}<0.001)$ (Table 1). Based on ORs, factors associated with discharge were age, $\mathrm{Hgb}, \mathrm{BUN}, \mathrm{Cr}$, albumin, ASA use, Oakland score and CCI (Table 5).

\section{Discussion}

In this cohort of patients presenting to the ED with overt LGIB, two-thirds of the cohort were admitted. However, outcomes including re-bleeding rates, GIB-associated re-admissions and bleeding-related mortality were not statistically different between the admitted and discharged groups, suggesting a potential role for outpatient management in these stable low-risk patients. The study demonstrated that those likely to be discharged were of younger age, particularly those under 50. Age, $\mathrm{Hgb}$, BUN, Cr, ASA use and CCI appear to be factors associated with discharge from the ED.

The Oakland score, which was validated in the UK as predictive of safe discharge with a score $\leq 8$ and a score as high as 10 in a US study, was applied to this cohort [7, 10]. Oakland et al defined safe discharge as lack of re-bleeding, red blood cell transfusion, therapeutic intervention, in-hospital death and GIB-related readmission within 28 days. In this cohort, 
Table 3. Comparison of Characteristics Between Discharged Patients With LGIB That Followed Up Compared to Those Lost to Follow Up

\begin{tabular}{|c|c|c|c|c|c|}
\hline & Totol & & Follow-up & & D wolue \\
\hline & Iotal & No & Yes & Unknown & 1-valute \\
\hline Age & $53(35-73)$ & $41.5(30.5-58.8)$ & $58(40-77)$ & $52(34.8-63.5)$ & 0.404 \\
\hline Under 50 & $16(43.2)$ & $5(62.5)$ & $5(29.4)$ & $6(50)$ & \\
\hline $50-59$ & $7(18.9)$ & $1(12.5)$ & $4(23.5)$ & $2(16.7)$ & \\
\hline $60-69$ & $3(8.1)$ & $1(12.5)$ & $1(5.9)$ & $1(8.3)$ & \\
\hline Over 80 & $5(13.5)$ & $1(12.5)$ & $3(17.6)$ & $1(8.3)$ & \\
\hline Race & & & & & 0.341 \\
\hline Asian & $5(13.5)$ & $0(0)$ & $1(5.9)$ & $4(33.3)$ & \\
\hline Black or African American & $4(10.8)$ & $2(25)$ & $2(11.8)$ & $0(0)$ & \\
\hline White & $14(37.8)$ & $3(37.5)$ & $7(41.2)$ & $4(33.3)$ & \\
\hline Gender (male) & $24(64.9)$ & $5(62.5)$ & $12(70.6)$ & $7(58.3)$ & 0.904 \\
\hline $\mathrm{CCI}$ & $2(0-3)$ & $0(0-3)$ & $3(1-4)$ & $0.5(0-3)$ & 0.131 \\
\hline Oakland score & $11(10-13)$ & $10(10-10.2)$ & $11(9-13)$ & $11(9.8-14)$ & 0.623 \\
\hline
\end{tabular}

Data are presented as frequency (\%) or median (IQR). P-value is calculated from a Kruskal-Wallis test for a numeric variable and a Fisher's exact test for a categorical variable. CCl: Charlson Comorbidity Index.

Table 4. Comparison of Outcomes Between Discharged and Admitted Patients With LGIB



Data are presented as frequency (\%) or median (IQR). P-value is calculated from a Wilcoxon rank-sum test for a numeric variable and a Fisher's exact test for a categorical variable. *Statistical significance with a P-value less than 0.05 . LGIB: lower gastrointestinal bleeding; ICU: intensive care unit. 
Table 5. Association Between Demographic and Clinical Characteristics and Discharge Status Among Patients With LGIB

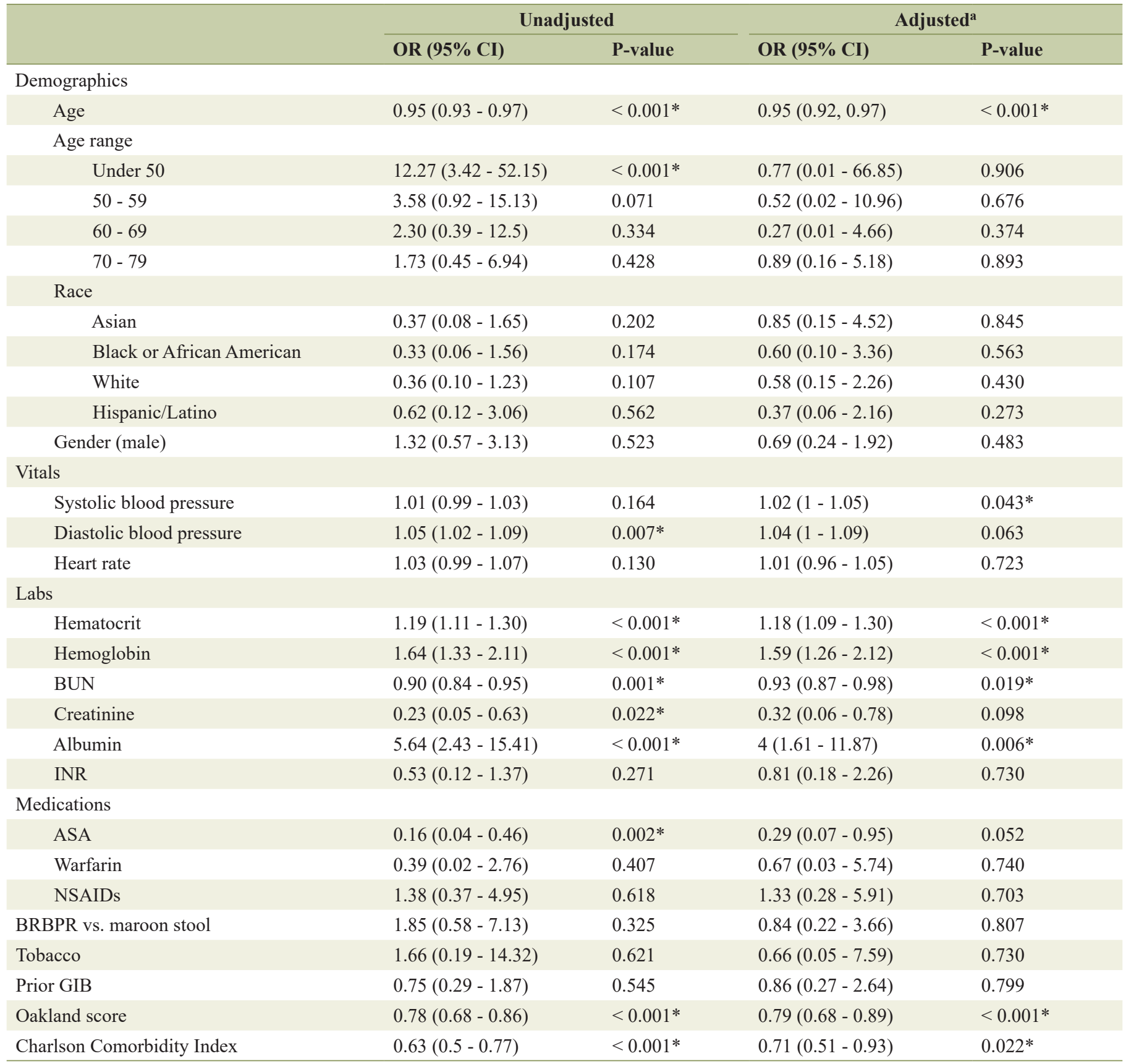

${ }^{a}$ Adjusted for age, race and gender. *Statistical significance with a P-value less than 0.05. LGIB: lower gastrointestinal bleeding; BUN: blood urea nitrogen; INR: international normalized ratio; ASA: aspirin; NSAIDs: nonsteroidal anti-inflammatory drugs; BRBPR: bright red blood per rectum; GIB: gastrointestinal bleeding; OR: odds ratio; $\mathrm{Cl}$ : confidence interval.

discharged patients had a statistically significant lower Oakland score of 11 with $51 \%$ of the patients having an Oakland $>$ 10. None of the discharged patients required transfusion during their evaluation, $14 \%$ had recurrent bleeds within 30 days and $11 \%$ were readmitted for recurrent bleed. However, when compared to those admitted, there were no significant 30-day bleeding-related deaths or all-cause mortality and these rates where comparative to prior studies in the general population (30-day re-bleeding 6-17\%; readmission 4-13\%; 30-day mortality $0-9 \%)[5,7,9,11-13]$. This suggests that stable LGIB patients could be safely discharged and an Oakland score with a threshold even higher than 8 could likely be applied to select those that are at low risk of adverse outcomes. Notably, the Oakland score studies included all LGIB patients while our study specifically focused on stable overt GI bleeders. Based on our study, we propose that even with an Oakland score of $>$ 
8, if the $\mathrm{SBP}>100 \mathrm{~mm} \mathrm{Hg}, \mathrm{HR}<100$ and the $\mathrm{Hgb}$ is above 13 $\mathrm{g} / \mathrm{dL}$, safe discharge with outpatient management may still be appropriate. We also recommend considering comorbid conditions, BUN/Cr and the use of blood thinners in the decision of whether outpatient management is appropriate. However, larger prospective studies are needed to further validate this, particularly including the role of anticoagulation and anti-platelet agents in our ability to predict safe discharge.

A major critique of the Oakland score was the inability to account for coagulation due to the lack of INR values in $38 \%$ of patients. While $95 \%$ of our cohort had an INR on presentation and was found to have no statistically significant association with discharge (OR $0.81, \mathrm{P}=0.730)$, this sample size was small and specific to patients presenting only with hemodynamically stable bleeding, thus limiting our ability to make generalizable predictions on its role in safe discharge for all LGIB patients. However, as all patients on DOAC were admitted as well as $80 \%$ of those on warfarin, anticoagulation seems to sway providers towards inpatient management. While ASA use was four times higher in those admitted, when adjusted for age, it was not predictive of discharge status. It is possible that older patients were more likely to be on ASA and were also more likely to be admitted presumably due to increased comorbid conditions. Nevertheless, other studies have demonstrated similar trends with anticoagulation and antiplatelet therapy being associated with a two- to fourfold increase in admissions for LGIB [6]. Ultimately, the safety of discharging stable patients on anticoagulation needs further evaluation with larger prospective studies.

In our study, $37 \%$ of patients underwent colonoscopy or flexible sigmoidoscopy, of which $17 \%$ resulted in therapeutic intervention compared to a range of $2-34 \%$ in the general population, particularly with higher rates of therapy with early vs. elective colonoscopy $[4,14]$. All of the patients who underwent endoscopic intervention were inpatients with average time to procedure of 2.8 days. While earlier colonoscopy is more likely to yield therapeutic outcomes, the overall rate of intervention is still relatively low. Cost-effective analysis is needed to further evaluate the benefit of this approach especially as the median LOS for inpatient management is 2 days with no statistical difference in adverse outcomes when compared to the discharged group.

In addition to adverse outcomes, a clear discharge follow-up plan is critical to assessing safety of ED discharge in patients with LGIB. Consistent with other studies, our data demonstrated that in most cases the etiology of LGIB is from benign pathologies such as diverticulosis, hemorrhoids and colonic ulcers. However, studies have demonstrated a $2-15 \%$ rate of finding colon neoplasm in patients presenting with lower GIB $[15,16]$. As a result, it is important to ensure appropriate outpatient evaluation to exclude malignant causes, particularly given the increased incidence of colon cancer in younger age groups, as younger age was shown to be predictive of discharge [17]. In our study, of the 36 patients discharged from the ED, only $8 \%$ received an appointment prior to discharge, $19 \%$ were contacted with an appointment by a patient navigator after discharge and $72 \%$ were responsible for making their own follow-up appointment with a PCP or a gastroenterologist. Among those discharged, 46\% followed up with a PCP, gastroenterologist or colorectal surgeon postdischarge for GI bleeding, with $91 \%$ of those visits occurring within 30 days of discharge. Eight outpatient colonoscopies were performed with primarily diverticular and hemorrhoidal disease. The lost to follow-up rate was $54 \%, 32 \%$ of whom had no further contact within our healthcare system but could have possibly been seen at other institutions. There was no difference in age, race or gender amongst patients that followed up and those that did not. While avoiding unnecessary hospitalizations may result in more appropriate allocation of inpatient resources, reduced LOS and a reduction in overall healthcare cost, it is imperative that health systems employ effective mechanisms for timely outpatient evaluation. Providing patients with an outpatient appointment at the time of discharge may increase this follow-up rate. Though recommendations on the exact follow-up interval is unclear, the British guidelines and our study suggest that 2 - 4 weeks postdischarge, depending on the severity of the bleed, seems to be safe [18].

While our study is the first to evaluate post-discharge follow-up and compare characteristics of admitted and discharged patients with stable LGIB, there are limitations when interpreting our results. Data were collected retrospectively and the decision for admission or discharge is not solely based on clinical parameters but likely reliant on unmeasured biases such as personal subjectivity and the clinical judgement of each individual provider on the clinical scenario. We also relied heavily on providers for documentation of bleeding and thus milder cases including those with no documented rectal exam may have been missed. The rectal exam is a key physical finding that suggests the location and severity of bleeding and can help guide management decisions. In our cohort, all patients had confirmed evidence of rectal bleeding on exam which in prior studies was unable to be assessed [10]. Nevertheless, misclassification of bleeding sources could have occurred since $62 \%$ of patients did not undergo endoscopy and maroon stools or hematochezia could sometimes represent an upper GIB etiology. The sample size of the study was also small and selected in an urban academic center which limits the generalizability to the overall population. One of the biggest limitations is the number of patients lost to follow-up which could have resulted in an under-estimation of our outcome measures. Chart review was limited to within our health system, and therefore this significantly impacted observed rates of follow-up in our study. Nevertheless, this study suggests a potential for optimizing our management of LGIB with the possibility for safe and early discharge in low-risk patients who may otherwise be unnecessarily hospitalized.

\section{Conclusion}

In hemodynamically stable patients with no other indication for hospital admission, there is considerable uncertainty regarding the optimal management of LGIB. While our findings support that early discharge in low-risk patients is probably safe and potentially cost-effective with a significantly reduced LOS, our current mechanism for outpatient follow-up remains suboptimal. Further prospective studies are needed to create 
evidence-based guidelines on appropriate timing and implementation of outpatient evaluation in these patients with stable but overt LGIB.

\section{Acknowledgments}

None to declare.

\section{Financial Disclosure}

None to declare.

\section{Conflict of Interest}

All authors declare that they have no conflict of interest to disclose.

\section{Informed Consent}

Not applicable.

\section{Author Contributions}

All authors contributed to the study concept and design, acquisition of data, analysis and interpreting data, drafting the manuscript, critical revision of the manuscript for important intellectual content and statistical analysis.

\section{Abbreviations}

LGIB: lower gastrointestinal bleeding; ED: emergency department; ACG: American College of Gastroenterology; LOS: length of stay; USA: United States of America; GI: gastrointestinal; GIB: gastrointestinal bleeding; SBP: systolic blood pressure; HR: heart rate; EGD: esophagogastroduodenoscopy; CCI: Charlson Comorbidity Index; IQR: interquartile range; OR: odds ratio; CI: confidence interval; DOAC: direct oral anticoagulant; INR: international normalized ratio; Hgb: hemoglobin; APC: argon plasma coagulation; CTA: computed tomography angiography; $\mathrm{PCP}$ : primary care provider; $\mathrm{BUN}$ : blood urea nitrogen; Cr: creatinine; ASA: aspirin; ICU: intensive care unit

\section{References}

1. Peery AF, Crockett SD, Murphy CC, Lund JL, Dellon ES, Williams JL, Jensen ET, et al. Burden and cost of gastrointestinal, liver, and pancreatic diseases in the United States: update 2018. Gastroenterology. 2019;156(1):254272 e211.

2. Strate LL, Gralnek IM. ACG clinical guideline: manage- ment of patients with acute lower gastrointestinal bleeding. Am J Gastroenterol. 2016;111(4):459-474.

3. Nagata N, Niikura R, Sakurai T, Shimbo T, Aoki T, Moriyasu S, Sekine K, et al. Safety and effectiveness of early colonoscopy in management of acute lower gastrointestinal bleeding on the basis of propensity score matching analysis. Clin Gastroenterol Hepatol. 2016;14(4):558-564.

4. Kouanda AM, Somsouk M, Sewell JL, Day LW. Urgent colonoscopy in patients with lower GI bleeding: a systematic review and meta-analysis. Gastrointest Endosc. 2017;86(1):107-117 e101.

5. Niikura R, Nagata N, Yamada A, Honda T, Hasatani K, Ishii N, Shiratori Y, et al. Efficacy and safety of early vs elective colonoscopy for acute lower gastrointestinal bleeding. Gastroenterology. 2020;158(1):168-175 e166.

6. Ng KS, Nassar N, Soares D, Stewart P, Gladman MA. Acute lower gastrointestinal haemorrhage: outcomes and risk factors for intervention in 949 emergency cases. Int J Colorectal Dis. 2017;32(9):1327-1335.

7. Oakland K, Jairath V, Uberoi R, Guy R, Ayaru L, Mortensen N, Murphy MF, et al. Derivation and validation of a novel risk score for safe discharge after acute lower gastrointestinal bleeding: a modelling study. Lancet Gastroenterol Hepatol. 2017;2(9):635-643.

8. Oakland K, Chadwick G, East JE, Guy R, Humphries A, Jairath V, McPherson S, et al. Diagnosis and management of acute lower gastrointestinal bleeding: guidelines from the British Society of Gastroenterology. Gut. 2019;68(5):776-789.

9. Oakland K, Kothiwale S, Forehand T, Jackson E, Bucknall C, Sey MSL, Singh S, et al. External validation of the Oakland score to assess safe hospital discharge among adult patients with acute lower gastrointestinal bleeding in the US. JAMA Netw Open. 2020;3(7):e209630.

10. Quan H, Li B, Couris CM, Fushimi K, Graham P, Hider $\mathrm{P}$, Januel JM, et al. Updating and validating the Charlson comorbidity index and score for risk adjustment in hospital discharge abstracts using data from 6 countries. Am J Epidemiol. 2011;173(6):676-682.

11. Oakland K, Guy R, Uberoi R, Hogg R, Mortensen N, Murphy MF, Jairath V, et al. Acute lower GI bleeding in the UK: patient characteristics, interventions and outcomes in the first nationwide audit. Gut. 2018;67(4):654662 .

12. Albeldawi M, Ha D, Mehta P, Lopez R, Jang S, Sanaka MR, Vargo JJ. Utility of urgent colonoscopy in acute lower gastro-intestinal bleeding: a single-center experience. Gastroenterol Rep (Oxf). 2014;2(4):300-305.

13. Siddique SM, Mehta SJ, Lewis JD, Neuman MD, Werner RM. Rates of hospital readmission among medicare beneficiaries with gastrointestinal bleeding vary based on etiology and comorbidities. Clin Gastroenterol Hepatol. 2019;17(1):90-97 e93.

14. Sengupta N, Tapper EB, Feuerstein JD. Early versus delayed colonoscopy in hospitalized patients with lower gastrointestinal bleeding: a meta-analysis. J Clin Gastroenterol. 2017;51(4):352-359.

15. Gralnek IM, Ron-Tal Fisher O, Holub JL, Eisen GM. The role of colonoscopy in evaluating hematochezia: a pop- 
ulation-based study in a large consortium of endoscopy practices. Gastrointest Endosc. 2013;77(3):410-418.

16. Ron-Tal Fisher O, Gralnek IM, Eisen GM, Williams JL, Holub JL. Endoscopic hemostasis is rarely used for hematochezia: a population-based study from the Clinical Outcomes Research Initiative National Endoscopic Database. Gastrointest Endosc. 2014;79(2):317-325.

17. Bailey CE, Hu CY, You YN, Bednarski BK, RodriguezBigas MA, Skibber JM, Cantor SB, et al. Increasing dis- parities in the age-related incidences of colon and rectal cancers in the United States, 1975-2010. JAMA Surg. 2015;150(1):17-22.

18. National Collaborating Centre for Cancer. National Institute for health and care excellence: clinical guidelines. Suspected cancer: recognition and referral. London: National Institute for Health and Care Excellence (UK). Copyright (C) National Collaborating Centre for Cancer; 2015. 